\title{
SNP and gene networks construction and analysis from classification of copy number variations data
}

\author{
Yang Liu, Yiu Fai Lee, Michael $\mathrm{K} \mathrm{Ng}^{*}$ \\ From IEEE International Conference on Bioinformatics and Biomedicine 2010 \\ Hong Kong, PR China. 18-21 December 2010
}

\begin{abstract}
Background: Detection of genomic DNA copy number variations (CNVs) can provide a complete and more comprehensive view of human disease. It is interesting to identify and represent relevant CNVs from a genomewide data due to high data volume and the complexity of interactions.

Results: In this paper, we incorporate the DNA copy number variation data derived from SNP arrays into a computational shrunken model and formalize the detection of copy number variations as a case-control classification problem. More than $80 \%$ accuracy can be obtained using our classification model and by shrinkage, the number of relevant CNVs to disease can be determined. In order to understand relevant CNVs, we study their corresponding SNPs in the genome and a statistical software PLINK is employed to compute the pair-wise SNPSNP interactions, and identify SNP networks based on their $P$-values. Our selected SNP networks are statistically significant compared with random SNP networks and play a role in the biological process. For the unique genes that those SNPs are located in, a gene-gene similarity value is computed using GOSemSim and gene pairs that have similarity values being greater than a threshold are selected to construct gene networks. A gene enrichment analysis show that our gene networks are functionally important.

Experimental results demonstrate that our selected SNP and gene networks based on the selected CNVs contain some functional relationships directly or indirectly to disease study.
\end{abstract}

Conclusions: Two datasets are given to demonstrate the effectiveness of the introduced method. Some statistical and biological analysis show that this shrunken classification model is effective in identifying CNVs from genomewide data and our proposed framework has a potential to become a useful analysis tool for SNP data sets.

\section{Background}

Copy number variation (CNV) is defined as a genomic segment range from one kilobase to several megabases in size, in which copy number differences have been observed by comparison of two or more reference genomes $[1,2]$. Human beings ordinarily have two copies of each autosomal region, one per chromosome. CNVs can be caused by genomic rearrangements such as inversions, deletions and duplications.

\footnotetext{
* Correspondence: mng@hkbu.edu.hk

Department of Mathematics, Hong Kong Baptist University, Kowloon Tong, Hong Kong

Full list of author information is available at the end of the article
}

The fact that DNA copy number variation is a widespread and common phenomenon among human beings was first discovered $[3,4]$ following the completion of the human genome project. The high variability of the copy number throughout the human genome have been found by investigating on 270 HapMap individuals [5,6]. Various studies have been developed for genome-wide $\mathrm{CNV}$ analysis to suggest their significant role in understanding human genetics. Redon et al. [7] published the first comprehensive and global map of CNVs in a genome-wide scale for making the beginning of large-scale copy number analysis. Comparative genomic hybridization $(\mathrm{CGH})[8]$ and array-based comparative genomic
C Biomed Central

() 2011 Liu et al; licensee BioMed Central Ltd. This is an open access article distributed under the terms of the Creative Commons Attribution License (http://creativecommons.org/licenses/by/2.0), which permits unrestricted use, distribution, and reproduction in any medium, provided the original work is properly cited. 
hybridization [9] can detect gains and losses of genomic segments. Based on CGH, Wang et al. [10] proposed a new algorithm "Cluster Along Chromosomes" (CLAC), which builds hierarchical clustering-style trees along each chromosome and selects the interesting clusters by controlling False Discovery Rate (FDR) at a certain level. Bayesian model is a popular method for CNV detection. Pique-Regi et al. [11] exploited the use of piecewise constant (PWC) vectors to represent genome copy number and sparse Bayesian learning (SBL) to detect CNA breakpoints. Wu et al. [12] implemented Bayesian segmentation approach to carry out segmentation and assigning copy number status simultaneously. Recently, Chen et al. [13] used the mean and variance change point model (MVCM) to detect CNVs or breakpoints with an approximate $p$-value for statistical testing. In addition, Oldridge et al. [14] quantitatively evaluated several processing and segmentation strategies when short-sequence oligonucleotide arrays are applied and provide guidelines to optimize performance based on study-specific objectives.

Besides the above mentioned CNV detection methods, high-density single nucleotide polymorphism (SNP) arrays are becoming more and more popular in $\mathrm{CNV}$ detection. The main reason is that the inheritance pattern and linkage disequilibrium of CNVs are similar to those of the SNPs [6,7]. Zhao et al. [15] measured the locus-specific hybridization intensity by hybridizing genomic representations of both DNA to SNP arrays to detect some novel CNVs in cancer samples. Huang et al. [16] proposed an algorithm that used whole genome sampling analysis (WGSA) by jointly measuring perfect match intensity and discrimination ratios to identify copy number changes. An QuantiSNP algorithm [17] described in 2007 used Objective Bayes Hidden-Markov Model (OB-HMM) by incorporating $\log \mathrm{R}$ ratio and $\mathrm{B}$ allele frequency to set certain hyperparameters as priors, and using a novel re-sampling framework to calibrate the model to a fixed false positive error rate for CNVs detection. Wang et al. [18] designed a tool named PennCNV, a hidden Markov model (HMM) based approach, for kilobase-resolution detection of CNVs by combining multiple sources of information together, not only the total signal intensity and allelic intensity ratio of each SNP marker, but also the neighboring distances, the allele frequency and the pedigree information.

Recently, disease classification using copy number variation data has been demonstrated by several groups [19-22]. The motivation of this kind of method is that appropriate machine learning models for disease classification using copy number variation data will be effective not only for clinical treatment, but also for identification of disease susceptibility loci. Generally, copy numbers at probe loci are used directly as features. In a CNV data set, the association between a disease and a set of relevant $\mathrm{CNVs}$ are investigated. Patients and normals are often categorized in groups according to their copy number changes. Thousands of CNVs in different regions of chromosomes are used to describe characteristics of patient/normal samples.

When many CNVs are used to detect the association between a disease and multiple marker genotypes, we expect in a typical data set that contains the CNV data of several thousands of CNVs in different individuals, it is common to find only several numbers of copy number positions having genetic patterns that are highly specific to each group of individuals. The CNVs are called the relevant CNVs, as opposed to the irrelevant CNVs that do not help much in identifying the group (i.e., individuals of the same type). Due to the large number of CNVs being irrelevant to each group, two individuals in the same group could have low similarity when measured by a simple similarity function that consider the characteristics of all CNVs. The groups may thus be undetectable by classification algorithms. Here, we are interested in the development of high-dimensional numerical classification algorithm that can identify group of individuals and their relevant $\mathrm{CNVs}$, i.e., detect the association between a disease and multiple marker variations. In this paper, we address this problem by applying a shrunken dissimilarity measure to copy number variation values derived from genome-wide SNP genotyping data. Performance was measured via crossvalidation classification accuracy. By shrinkage, the number of relevant CNVs can be determined. In order to understand relevant CNVs, we study their corresponding SNPs in the genome and a statistical software PLINK is employed to compute the pair-wise SNP-SNP interactions, and identify SNP network based on their $P$-values. Some statistical analysis are done to illustrate the significance of our selected SNP networks. For the unique genes that those SNPs are located in, a genegene similarity value is computed using GOSemSim and gene pairs that has a similarity value being greater than a threshold are selected to construct gene networks. A gene enrichment analysis are done to show that some molecular functions and biological process are significantly associated with our gene networks. The whole framework indicates that our classification model is efficient in high dimensional CNV detection and SNP and gene networks constructed afterwards have relationships directly or indirectly to disease study.

The outline of this paper is given as follows. In Section 2, we propose the LRR calculation for signal intensities, the shrunken dissimilarity measure to analyze $\mathrm{CNV}$ data classification and the logistic model to calculate SNP interactions. In Section 3, we present experimental results on two real CNV data sets derived from 
genome-wide SNP arrays, and illustrate their corresponding SNP and gene networks for statistical and biological analysis. Finally, we give concluding remarks in Section 4.

\section{Methods}

\section{LRR calculation}

There are many available tools that can be used to do genotyping call and Log R Ratio (LRR) calculation, such as Birdsuite [23], CNAG [24], dChip [25,26] and GLAD [27]. We calculated LRR values using PennCNV [18], which is a public available software for copy number variation detection from SNP genotyping arrays. For each SNP in a genome-wide data, the raw signal intensity values for its two alleles $\mathrm{A}$ and $\mathrm{B}$ are measured and then subject to a normalization procedure. The normalized signal intensity values should reveal three clusters $(\mathrm{AA}, \mathrm{AB}, \mathrm{BB})$ representing three distinct genotypes. A genotype calling is a genotype assignment based on these three canonical clusters.

The normalization procedure produces $X$ and $Y$ values for each SNP, representing the experiment-wide normalized signal intensity on alleles $\mathrm{A}$ and $\mathrm{B}$, respectively. One additional measurement will then be calculated for each SNP, where $R=X+Y$ refers to the total signal intensity. As for a normalized measure of total signal intensity, the LRR value for each SNP is then calculated as

$$
L R R=\log _{2}\left(R_{\text {observed }} / R_{\text {expected }}\right)(1)
$$

where $R_{\text {expected }}$ is computed from linear interpolation of canonical genotype clusters [28]. LRR refers to the logarithm (in base 2) of the total observed normalized intensity $R_{\text {observed }}$ of the two alleles $A$ and $B$ relative to the expected $R_{\text {expected }}$. For normal diploid genotypes, LRR should fluctuate randomly around zero.

\section{Shrunken centroid method}

It is well-known that DNA microarray data, which can simultaneously measure the expression level of thousands of different genes, have been successfully used to identify genetic heterogeneity of disease. However, mircoarray data typically has a large number of genes (features) and relatively few samples (observations), meaning that conventional machine learning methods may fail when applied to such data. The Prediction Analysis for Microarrays (PAM), has recently been reported as a potential powerful tool for microarrays analysis, which is based on the technique of nearest shrunken centroid $[29,30]$. Nearest shrunken centroid is the "denoised" version of simple nearest centroid classification. The main idea of nearest centroid classification is to compare test sample to each class centroid. The class whose centroid the test sample is closest to is the predicted class for test sample. The centroid is the average value for each feature (or attribute) in each class, normalized by the pooled within-class standard deviation. The nearest shrinkage centroid classification shrinks each centroid toward the overall centroid for all classes by a certain amount, which is called a "threshold". After the overall centroid is set to zero, this shrinkage is to move the centroid to zero by the threshold, i.e., if the value of centroid in magnitude is larger than the threshold, it is subtracted by the threshold; if it is less than the threshold, it is set to zero, so the corresponding feature disappears, and it isn't used for the following classification. By doing so, nearest shrunken centroid can find out the minimal subset of genes which succinctly characterize each class. Shrunken centroid method is traditionally used to deal with microarray data. In this paper, we adopt this method for copy number variation values derived from SNP genotyping arrays. The normalized signal intensity Log R Ratio (LRR) value in each probe loci calculated from existing software, which we will give a detailed description in the next subsection, will be considered as a unique feature.

Suppose there are $n$ samples, $p$ CNVs, and $K$ classes. For $i$ th CNV, the class centroid $\bar{x}_{i j}$ is the average value of LRR values within one class, and the overall centroid $\bar{x}_{i}$ is the average value over all classes. The difference between class centroid and overall centroid is normalized by pooled with-class standard deviation as follows:

$$
d_{i x}=\frac{\bar{x}_{i k}-\bar{x}_{i}}{m_{k}\left(s_{i}+s_{0}\right)}
$$

where

$$
s_{i}^{2}=\frac{1}{n-K} \sum_{k=1}^{K} \sum_{j \in C_{k}}\left(x_{i j}-\bar{x}_{i k}\right)^{2}, \quad m_{k}=\sqrt{\frac{1}{n_{k}}-\frac{1}{n}}
$$

$C_{k}$ denote the indices of the $n_{k}$ samples in class $k$, and $s_{0}$ is a positive constant included to prevent the possibility that a CNV with a low LRR level could produce a large $d_{i j}$. Equation (2) can be rewritten as:

$$
\bar{x}_{i k}=\bar{x}_{i}+m_{k}\left(s_{i}+s_{0}\right) d_{i k}
$$

Then the shrunken centroid is defined as:

$$
\bar{x}_{i k}^{\prime}=\bar{x}_{i}+m_{k}\left(s_{i}+s_{0}\right) d_{i k}^{\prime}
$$

where $d_{i k}^{\prime}=\operatorname{sign}\left(d_{i k}\right)\left(\left|d_{i k}\right|-\Delta\right)_{+}$, and $t_{+}=t$ if $t>0$ and zero otherwise. The parameter $\Delta$ is usually sought by cross-validation with minimal classification error rate. Note that if $d_{i k}^{\prime}=0$ for all $k$ for a given $i$, then all of the shrunken centroids are zero, and the $i$ th $\mathrm{CNV}$ does not contribute to the classification process. For 
prediction, the class label of test sample $\mathbf{t}$ is determined by the nearest shrunken centroid:

$$
C(\mathbf{t})=\arg \min _{k}\left(\sum_{i} \frac{\left(t_{i}-\bar{x}_{i k}^{\prime}\right)^{2}}{\left(s_{i}+s_{0}\right)^{2}}-2 \log \left(\pi_{k}\right)\right), i \in\left\{i|| d_{i k} \mid>\Delta\right\}
$$

where

$$
\delta_{k}(\mathbf{t})=\sum_{i \in\left\{i \mid d_{i k}>\Delta\right\}} \frac{\left(t_{i}-\bar{x}_{i k}^{\prime}\right)^{2}}{\left(s_{i}+s_{0}\right)^{2}}-2 \log \left(\pi_{k}\right)
$$

$\pi_{k}$ is the prior probability of class $\mathrm{k}$.

\section{Logistic model to calculate SNP interactions}

As our experimental data sets are both disease-trait samples, it is feasible to test epistasis using PLINK to [31] detect SNP-SNP interactions, which is a free, open-source whole genome association analysis toolset, designed to perform a range of basic, large-scale analysis in a computationally efficient manner. All pairwise combinations of input SNPs can be tested using a logistic regression model, which is based on allele dosage for each SNP, A and $\mathrm{B}$, and fits the model in the form of (5)

$$
Y \sim b_{0}+b_{1} \cdot A+b_{2} \cdot B+b_{3} \cdot A B+e(5)
$$

The test for interaction is based on the coefficient of $b_{3}$, therefore only considers allelic by allelic epistasis. We focus on symmetrical cases in our study, that means only unique pairs are analysed, for example, if SNP1"SNP2 is performed, SNP2*SNP1 will not be calculated again. The $\chi^{2}$ statistics is applied and the odds ratio for interaction is interpreted in the standard manner: a value of 1.0 indicates no effect.

\section{Results and Discussion \\ WTCCC Type 1 Diabetes study \\ Data set and preprocessing}

The core study of the Wellcome Trust Case Control Consortium (WTCCC) comprised an analysis of genetic signals from each of seven common human diseases (type 1 diabetes, type 2 diabetes, coronary artery disease, hypertension, bipolar disorder, rheumatoid arthritis and Croh disease) [32]. Genotyping for type 1 diabetes (T1D) study was conducted by Affymetrix using the ("commercial") Affymetrix 500K chip in 3504 samples with 2000 cases and 1504 controls.

The quantile normalized signal intensity data, which were generated from the Affymetrix intensity ('CEL') files and used as input to the Chiamo genotype calling program, was downloaded from WTCCC webpage and appropriately prepared according to the requirements of input file formats of PennCNV. A biological literature searching indicates that in chromosome 6 , there are some well-known T1D related genes. Therefore, we chose chromosome 6 as an example to demonstrate our method. There are 31470 unique SNP loci in this chromosome, 1 markers have complete genotyping failure with confidence threshold of 0.01, 1042 markers do not have at least two types of genotypes, 2460 markers have abnormal patterns, so there are 27967 SNP markers have been analyzed to construct canonical clustering positions.

\section{Classification results}

After a 10-fold cross validation setting, 3154 samples were selected as training data and the remaining 350 samples would be the testing data. The highest classification accuracy (correctly classified samples in testing data sets in the 10-fold cross validation) in the 10 trials is $80 \%$. The parameter $\Delta$ was tuned to obtain the highest accuracy in the test. Figure 1 shows the relationships between $\Delta$ values and accuracies obtained in this trial. We can observe that when the value of $\Delta$ is increased from 0 to 20, the classification accuracy fluctuates and achieves the highest one of $80.00 \%$ when $\Delta=10.56$.

Our method can select 63 SNPs, 25 of them are located in gene coding areas. In order to investigate how this small set of SNPs can distinguish case and control people so well, we further discovered the distribution of each SNP in case and control group. Table 1 shows the average and variance of LRR values in each SNP position within case and control group respectively. We only considered the SNPs that are located in gene coding areas here. We gave a ranking for these SNPs based on the parameter of $d_{i k}^{\prime}$ mentioned in the previous section, which can be considered as the contribution of this SNP to the classification problem. We can see that in each SNP position, the distribution of LRR values within case people and control people are significantly different, which are consistent with our classification results.

\section{SNP network construction and analysis}

All the SNPs selected by our method can be divided into two categories, those are located in gene coding areas and those are not. We did a statistical analysis between these two categories of SNPs using PLINK [31]. All pairwise combinations of SNPs can be tested. Odds ratio for interaction, $\chi^{2}$ statistic and asymptotic $P$-value will be provided in the output file. When adopted different thresholds, different kinds of SNP networks can be constructed. Here "threshold" means only SNP pairs that have a P-value smaller than this threshold will be considered and be included in the SNP network. By constructing these SNP networks, we can figure out some potential SNP-SNP interactions that are still unknown. Table 2 shows a detailed characteristics of SNP networks under different thresholds, including the number of SNP networks, the number of nodes and edges in each individual network. As we were interested 


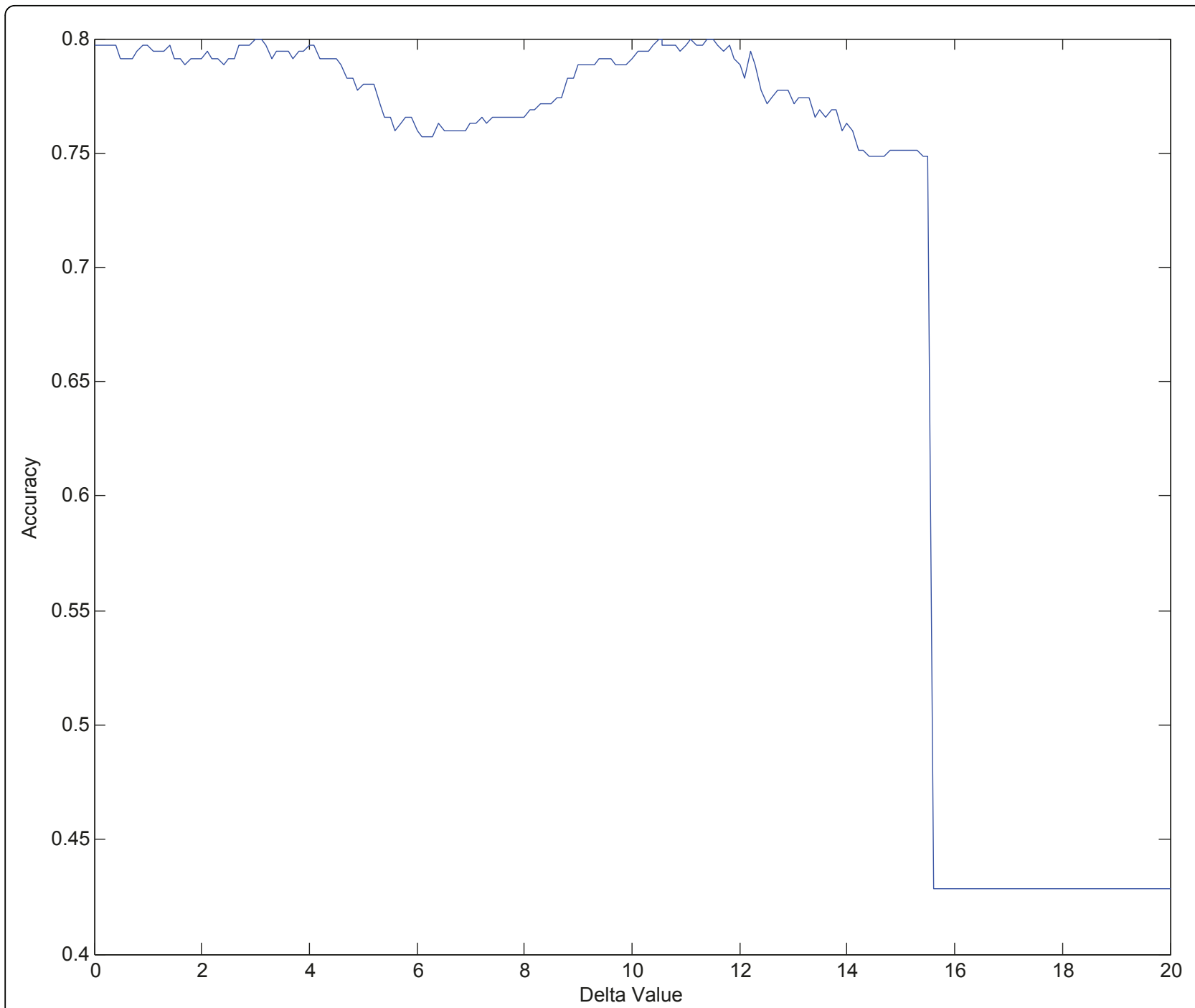

Figure 1 Relationship between $\Delta$ and accuracy in chromosome 6 of T1D study. Illustration of the accuracy obtained in chromosome 6 of Type 1 diabetes data set when change $\Delta$ value from 0 to 20. For chromosome 6, in each trial, all the 3504 samples of both control and case were randomly divided into 10 equal partitions. For each of the 10 partition groups, we selected one of them as testing set and the remaining nine of them were considered as training sets. 10 trials were considered and the results were collected based on this 10-fold cross validation procedure. This figure was drawn based on one of these ten trails when the highest accuracy (accuracy refers to the percentage of correctly classified samples over all test samples) was obtained. $X$ axis refers to $\Delta$ value, it increases from 0 to 20. $Y$ axis refers to the accuracy obtained in chromosome 6 when using our method, it fluctuates when different $\Delta$ values are applied and the highest accuracy is obtained when $\Delta$ is equal to 10.56 .

at SNP-SNP interactions, and we only considered the networks where the number of SNPs are more than one. We can see that the number of networks increases when the significant P-value decreases as one single network can be separated into several smaller ones. However, when P-value further decreases, the number of networks is reduced until there is only one left as there are too small SNPs to form a network.

To further utilize and benefit from these SNP networks selected by our method, we next performed some statistical analysis. For each network under one particular threshold, we randomly picked the same number of SNPs with our selected SNP network to get a random SNP network, calculated all the pairwise SNPSNP interactions and got the average value of all the Pvalues within this random network. Such process were repeated 500 times. Table 3 shows the significance of our selected networks compared with random ones. As SNP networks under threshold of 0.1 almost involve all the SNPs selected by our shrunken classification model, we chose this threshold as an example to further analyze. Figure 2 shows the two SNP networks under this 
Table 1 Distribution of LRR values for selected SNPs of T1D study.

\begin{tabular}{llllll}
\hline SNP ID & $d_{i k}$ & \multicolumn{2}{c}{ Control } & \multicolumn{2}{c}{ Case } \\
& & mean & variance & mean & variance \\
\hline rs6940177 & 2.3940 & 0.0522 & 0.0431 & -0.1845 & 0.0402 \\
rs17209874 & 2.2398 & 0.1071 & 0.0288 & -0.0926 & 0.0218 \\
rs9479373 & 2.1075 & 0.1027 & 0.0187 & -0.0838 & 0.0213 \\
rs3778077 & 1.8133 & 0.0937 & 0.0157 & -0.1180 & 0.0450 \\
rs16875181 & 1.6937 & 0.1107 & 0.0216 & -0.1033 & 0.0454 \\
rs7754428 & 1.6853 & 0.0968 & 0.0199 & -0.0921 & 0.0262 \\
rs6902158 & 1.6838 & 0.0888 & 0.0278 & -0.1067 & 0.0244 \\
rs6912853 & 1.3226 & 0.1116 & 0.0254 & -0.0770 & 0.0274 \\
rs991974 & 1.2833 & 0.0912 & 0.0177 & -0.1010 & 0.0351 \\
rs643394 & 1.2636 & 0.1146 & 0.0244 & -0.0943 & 0.0471 \\
rs9397339 & 1.1925 & 0.0906 & 0.0177 & -0.0906 & 0.0264 \\
rs319123 & 1.1473 & 0.1015 & 0.0199 & -0.0863 & 0.0309 \\
rs352095 & 0.9764 & 0.1097 & 0.0380 & -0.0873 & 0.0310 \\
rs1738262 & 0.7388 & 0.0979 & 0.0249 & -0.0860 & 0.0311 \\
rs7760230 & 0.5918 & 0.0914 & 0.0171 & -0.0730 & 0.0204 \\
rs10947885 & 0.4503 & 0.0663 & 0.0166 & -0.1074 & 0.0308 \\
rs1334689 & 0.4209 & 0.1052 & 0.0197 & -0.0775 & 0.0361 \\
rs1406882 & 0.3947 & 0.1047 & 0.0236 & -0.0837 & 0.0402 \\
rs1563666 & 0.2513 & 0.0916 & 0.0329 & -0.0846 & 0.0245 \\
rs9321142 & 0.2245 & 0.0843 & 0.0140 & -0.0719 & 0.0210 \\
rs2073012 & 0.1519 & 0.0989 & 0.0281 & -0.0668 & 0.0208 \\
rs11154452 & 0.1286 & 0.0847 & 0.0243 & -0.0905 & 0.0324 \\
rs9394755 & 0.1208 & 0.0980 & 0.0270 & -0.0714 & 0.0257 \\
rs9371491 & 0.0910 & 0.0832 & 0.0151 & -0.0732 & 0.0217 \\
rs6918886 & 0.0069 & 0.0951 & 0.0220 & -0.0922 & 0.0473 \\
\hline & & & & &
\end{tabular}

threshold with one is smaller, including only 2 SNPs, but the other one is much bigger, including 49 unique SNPs. Figure 3 is the cumulative distribution of the average P-values for all 500 samples (each with 49 SNPs). Compared with the distribution of random samples, which was with mean of 0.5645 and variance of 0.1008 , our selected SNP network (average P-value is 0.0450 ) is very significant, with significance value of $1.2765 \times 10^{-7}$.

\section{Gene network construction and analysis}

Our method can select 63 SNPs, 25 of them are located in gene coding areas, and these 25 SNPs belong to 24

Table 2 Characteristics of SNP networks in different thresholds of T1D study.

\begin{tabular}{llll}
\hline $\begin{array}{l}\text { PLINK } \\
\text { thresholds }\end{array}$ & $\begin{array}{l}\text { NO. of } \\
\text { networks }\end{array}$ & NO. of SNPs & NO. of SNP pairs \\
\hline 0.1 & 2 & $1(2), 2(49)$ & $1(1), 2(60)$ \\
0.05 & 4 & $1(3), 2(4), 3(9), 4(20)$ & $1(2), 2(3), 3(8), 4(21)$ \\
0.01 & 5 & $1(2), 2(2), 3(2), 4(2)$, & $1(1), 2(1), 3(1), 4(1)$, \\
& & $5(5)$ & $5(4)$ \\
0.005 & 3 & $1(2), 2(2), 3(5)$ & $1(1), 2(1), 3(4)$ \\
0.001 & 1 & $1(2)$ & $1(1)$ \\
\hline
\end{tabular}

unique genes, which are all shown in Table 4. After checking with NCBI, we found that some of the genes that our selected SNPs located in are directly or indirectly related to diabetes. For example, GMDS refers to GDP-mannose 4,6-dehydratase, which catalyzes the conversion of GDP-mannose to GDP-4-keto-6-deoxymannose, and it has been verified that mannose-binding lectin is a predictor of Type 1 Diabetes [33]. MTHFD1L is an enzyme involved in THF synthesis in mitochondria, and mutations of mitochondria strongly associate with diabetes [34]. And also for LAMA2, whose full name is laminin, alpha 2 , is a major component of the basement membrane, the abnormal level of it has been reported to have significant relationship to the presence of diabetes [35].

We computed all the pair-wise functional similarities of these 24 gene products using GOSemSim, which is an open source and open development software project for the analysis and comprehension of genomic data running in the platform of R Bioconductor package [36]. GOSemSim estimates the similarity scores of gene pairs according to their Gene Ontology (GO) [37] terms: molecular function (MF), biological process (BP) and cellular component (CC). In this paper, we only considered two of these terms: MF and BP and adopted Rel's method [38] to compute the similarity values, which is based on the information content of the GO terms and define information content as the frequency of each term occurs in the GO corpus. Afterwards, gene pairs that have a similarity value being greater than a threshold, were selected to construct a gene network using Cytoscape [39]. As we were interested at gene-gene interactions, and we only considered the networks where the number of genes are more than one. In Table 5, we showed the number of gene networks formed by using different threshold values and the number of pairs of genes involved.

We can see in Table 5 that the number of gene networks increases when the threshold value increases as more networks are formed. However, when threshold value further increases, the number of networks is reduced until there is only one network left. According to Table 5, we selected the threshold of 0.15 for analysis as the number of gene networks is highest than those using other threshold values. Figure 4 demonstrates the gene networks constructed by our method when threshold is equal to 0.15 . Gene pairs that are grouped in the same network suggested a strong potential for interaction effects in biological process. We can see from this figure that there are 3 networks, including 12 pairs and 11 unique genes.

We found some interesting relationships from SNP and gene networks. For example, for SNPs rs 9321142 and rs9371491, which are interacted in the same SNP network under $P$-value of 0.1 in Figure 2, their 
Table 3 Significance of selected SNP networks in different thresholds of T1D study.

\begin{tabular}{|c|c|c|c|c|c|}
\hline \multirow[t]{2}{*}{ PLINK thresholds } & \multirow[t]{2}{*}{ NO. of networks } & \multirow[t]{2}{*}{ Selected mean } & \multicolumn{2}{|c|}{ Random } & \multirow[t]{2}{*}{ Significance value } \\
\hline & & & mean & variance & \\
\hline \multirow[t]{2}{*}{0.1} & 2 & $1(0.0985)$ & $1(0.5648)$ & $1(0.0991)$ & $1.2672 \times 10-6$ \\
\hline & & $2(0.0450)$ & $2(0.5645)$ & $2(0.1008)$ & $1.2765 \times 10-7$ \\
\hline \multirow[t]{4}{*}{0.05} & 4 & $1(0.0298)$ & $1(0.5805)$ & $1(0.1018)$ & $3.1578 \times 10-8$ \\
\hline & & $2(0.0225)$ & $2(0.5682)$ & $2(0.0995)$ & $2.0741 \times 10-8$ \\
\hline & & $3(0.0306)$ & $3(0.5638)$ & $3(0.1005)$ & $5.6191 \times 10-8$ \\
\hline & & $4(0.0203)$ & $4(0.5633)$ & $4(0.1002)$ & $2.9939 \times 10-8$ \\
\hline \multirow[t]{5}{*}{0.01} & 5 & $1(0.0054)$ & $1(0.5862)$ & $1(0.1003)$ & $3.5062 \times 10-9$ \\
\hline & & $2(0.0057)$ & $2(0.5645)$ & $2(0.0983)$ & $6.5545 \times 10-9$ \\
\hline & & $3(0.0012)$ & $3(0.5622)$ & $3(0.0971)$ & $3.7898 \times 10-9$ \\
\hline & & $4(0.0011)$ & $4(0.5545)$ & $4(0.1009)$ & $2.0716 \times 10-8$ \\
\hline & & $5(0.0015)$ & $5(0.5528)$ & $5(0.1004)$ & $1.9979 \times 10-8$ \\
\hline \multirow[t]{3}{*}{0.005} & 3 & $1(0.0011)$ & $1(0.5327)$ & $1(0.1024)$ & $1.0436 \times 10-7$ \\
\hline & & $2(0.0012)$ & $2(0.5300)$ & 2(0.0998) & $5.8348 \times 10-8$ \\
\hline & & $3(0.0015)$ & $3(0.5762)$ & $3(0.1004)$ & $5.1991 \times 10-9$ \\
\hline 0.001 & 1 & $1(0.0001)$ & $1(0.5578)$ & $1(0.0950)$ & $2.1721 \times 10-9$ \\
\hline
\end{tabular}

corresponding genes are LAMA2 and MTHFD1L, but located in different gene networks in Figure 4, which means that maybe we can merge these two gene networks together, (shown with red dash line in Figure 4), and furthermore, can be consistent with existing biological functions of these two genes to diabetes. Another example, rs6912853 is interacted with rs2734990 under a very significant $\mathrm{P}$-value of $9.43 \times 10^{-5}$, but rs 2734990 is located in intergenic area and do not have a record in
Gene Ontology until now, maybe we can make use of rs6912853's gene information, BTN3A1, to further analyze the inner functions of rs2734990 and extend GO afterward.

In order to investigate the inner functions of the genes involved in our gene network in an ontology level, we performed the GO enrichment analysis by GOEAST [40], which is a web-based software toolkit for fast identification of underlining biological relevance of high-

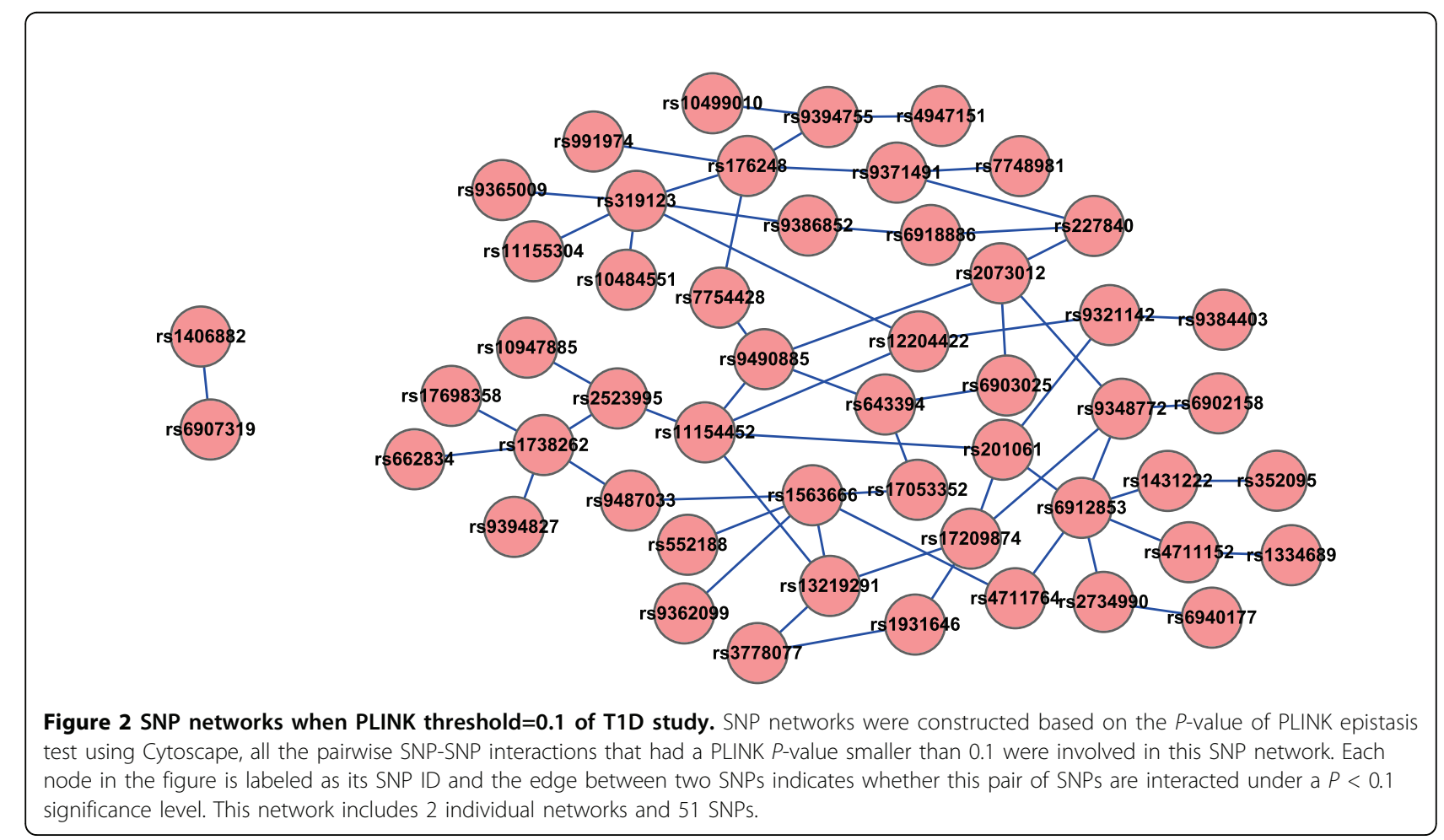




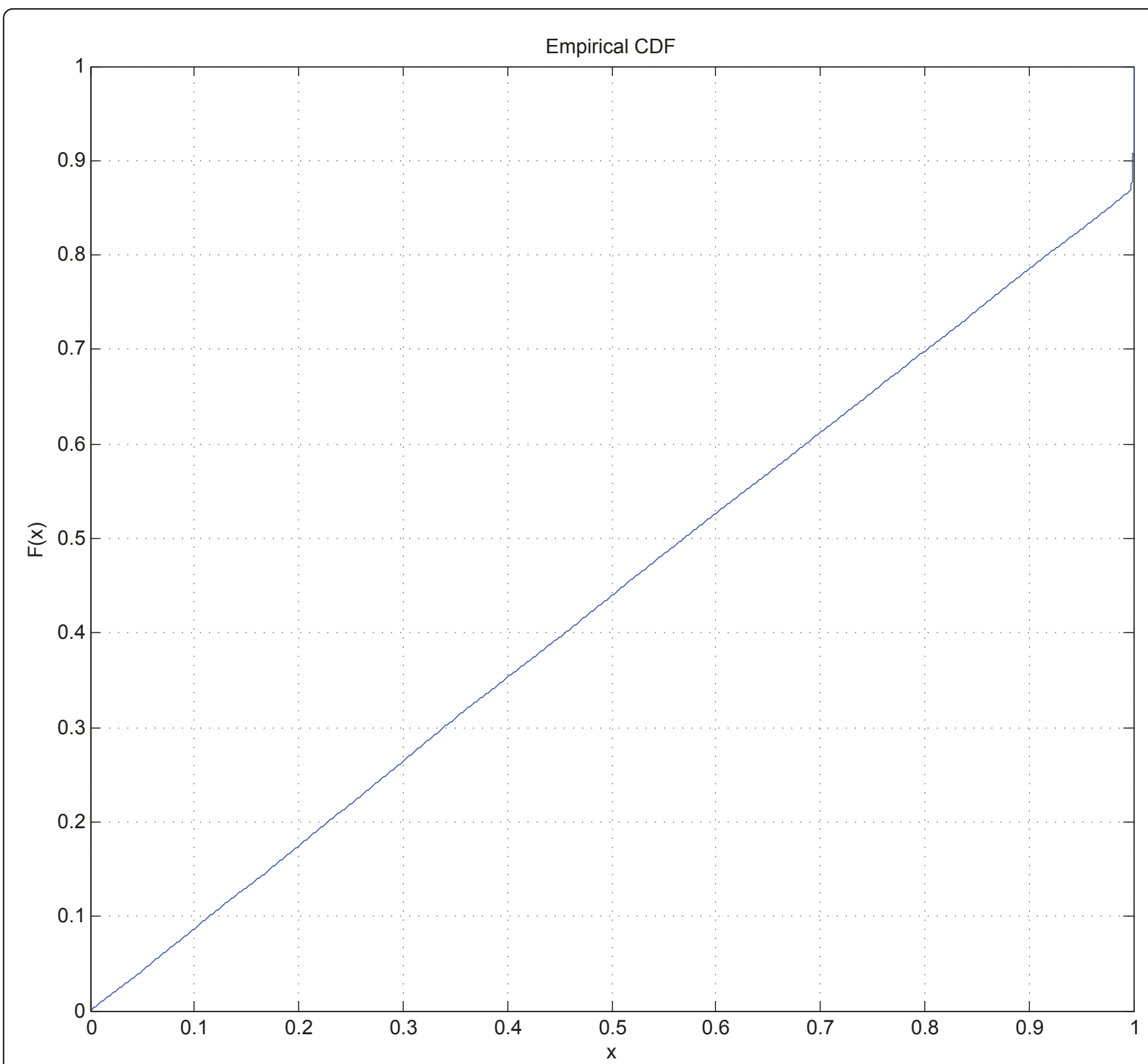

Figure 3 Cumulative distribution of $\mathbf{5 0 0}$ random SNP networks. Cumulative distribution of 500 random SNP networks. Each of these 500 random networks maintained the same size with our selected SNP network (49 SNPs). SNPs were selected randomly, calculated all pairwise SNPSNP interactions using PLINK, got the average P-value of all pairs. This cumulative distribution is a statistics of these 500 random networks with $X$ axis is the average $P$-value within this network and $Y$ axis is the cumulative probability that within a particular $X$ threshold.

throughput experimental results. GOEAST discovers statistically significantly enriched GO terms among the given gene list based on their hypergeometric probability. So the purpose of this analysis here is to find out which GO terms can be strongly enriched or significantly associated with our selected genes. We did a Batch-Genes search using "Homo sapiens" background for all the three GO categories: "molecular function", "biological process" and "cellular component", as shown in Figure 5, Figure 6 and Figure 7 respectively. In these figures, boxes represent GO terms, labeled by its GOID, term definition, $P$-value and detail information. Significantly enriched GO terms are marked yellow. The degree of color saturation of each node is positively correlated with the significance of enrichment of the corresponding GO term. The detail information labeled in the enriched GO nodes are organized as " $q / m \backslash t / k(p-$ value)", where $q$ is the count of genes associated with the listed GOID (directly or indirectly) in our dataset, $m$ is the count of genes associated with the listed GOID (directly or indirectly) on the chosen platform, $k$ is the total number of genes in our dataset, $t$ is the total 
Table 4 Detailed gene descriptions of SNPs selected in chromosome 6 of T1D study.

\begin{tabular}{|c|c|c|c|}
\hline SNP ID & $\begin{array}{l}\text { Gene } \\
\text { Symbol }\end{array}$ & $\begin{array}{l}\text { Gene } \\
\text { ID }\end{array}$ & Description \\
\hline rs6940177 & TRDN & 10345 & triadin \\
\hline rs17209874 & RUNX2 & 860 & runt-related transcription factor 2 \\
\hline rs9479373 & LOC646024 & 646024 & locus-region \\
\hline rs3778077 & NUDT3 & 11165 & nudix (nucleoside diphosphate linked moiety X)-type motif 3 \\
\hline rs16875181 & GPR116 & 221395 & G protein-coupled receptor 116 \\
\hline rs7754428 & GMDS & 2762 & GDP-mannose 4,6-dehydratase \\
\hline rs6902158 & LRRC16 & 55604 & leucine rich repeat containing 16 \\
\hline rs6912853 & BTN3A1 & 11119 & butyrophilin, subfamily 3 , member A1 \\
\hline rs991974 & LMBRD1 & 55788 & chromosome 6 open reading frame 209 \\
\hline rs643394 & LOC442256 & 442256 & similar to PPP1R14B protein \\
\hline rs9397339 & PLEKHG1 & 57480 & pleckstrin homology domain containing, family G (with RhoGef domain) member 1 \\
\hline rs319123 & C6orf210 & 57107 & chromosome 6 open reading frame 210 \\
\hline rs352095 & FLJ34503 & 285759 & hypothetical protein FLJ34503 \\
\hline rs1738262 & DNAH8 & 1769 & dynein, axonemal, heavy polypeptide 8 \\
\hline rs7760230 & SNX9 & 51429 & sorting nexin 9 \\
\hline rs10947885 & LRFN2 & 57497 & leucine rich repeat and fibronectin type III domain containing 2 \\
\hline rs1334689 & EPB41L2 & 2037 & erythrocyte membrane protein band 4.1 -like 2 \\
\hline rs1406882 & FYN & 2534 & FYN oncogene related to SRC, FGR, YES \\
\hline rs1563666 & LOC643281 & 643281 & intron \\
\hline rs9321142 & LAMA2 & 3908 & laminin, alpha 2 (merosin, congenital muscular dystrophy) \\
\hline rs2073012 & NOX3 & 50508 & NADPH oxidase 3 \\
\hline rs11154452 & LAMA2 & 3908 & laminin, alpha 2 (merosin, congenital muscular dystrophy) \\
\hline rs9394755 & UNC5CL & 222643 & unc-5 homolog C (C. elegans)-like \\
\hline rs9371491 & MTHFD1L & 25902 & $\begin{array}{l}\text { methylenetetrahydrofolate dehydrogenase (NADP+ dependent) 1-like an enzyme involved in THF synthesis in } \\
\text { mitochondria }\end{array}$ \\
\hline rs6918886 & RPS6KA2 & 6196 & ribosomal protein $\mathrm{S} 6$ kinase, $90 \mathrm{kDa}$, polypeptide 2 \\
\hline
\end{tabular}

number of genes on the chosen platform, $p$-value is of the significance for the enrichment in the dataset of the listed GOID under hypergeometric distribution. We also extracted all the $\mathrm{GO}$ terms and the corresponding involved genes with a significant $P<0.001$, see Table 6 . We highlighted the GO terms that may be related to diabetes, which need to be further verified and investigated by biologists.

\section{Hirschsprung study}

\section{Data set and preprocessing}

Hirschsprung (HSCR, MIM 142623), also known as aganglionic megacolon, is a congenital intestinal disease.

Table 5 Gene networks formed for different threshold values of T1D study.

\begin{tabular}{ccc}
\hline $\begin{array}{c}\text { GOSemSim } \\
\text { thresholds }\end{array}$ & $\begin{array}{c}\text { Number of gene } \\
\text { pairs }\end{array}$ & $\begin{array}{c}\text { Number of gene } \\
\text { networks }\end{array}$ \\
\hline 0.20 & 1 & 1 \\
0.19 & 11 & 2 \\
$0.15-0.18$ & 12 & 3 \\
0.14 & 14 & 2 \\
0.13 & 24 & 1 \\
\hline
\end{tabular}

Patients suffer from different extent of aganglionosis due to the absence of ganglion cells in the gastrointestinal tract. Trisomy 21 (Down syndrome) is the most recurrent structural variations found in HSCR, contributing to $2-10 \%$ of the cases.

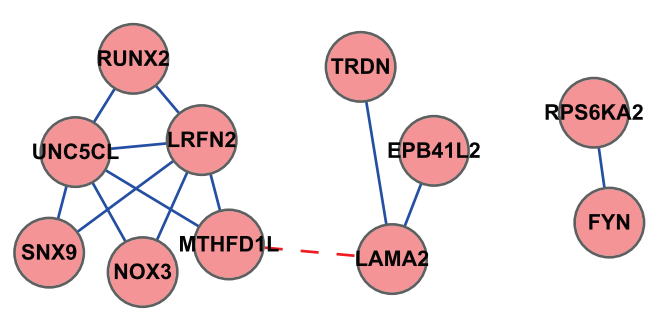

Figure 4 Gene network when GOSemSim threshold $=0.15$ of T1D study. Gene network constructed using Cytoscape. In chromosome 6 of T1D data, all SNPs selected belong to 24 unique genes. All the pairwise similarity values of these 24 genes were computed using GOSemSim and gene pairs that had a $>0.15$ threshold were grouped together. Every node in the figure is labeled as its gene symbol and the edge between two genes indicates whether this pair of genes has a $>0.15$ threshold or not. This network includes 3 individual networks and totally 11 genes. 


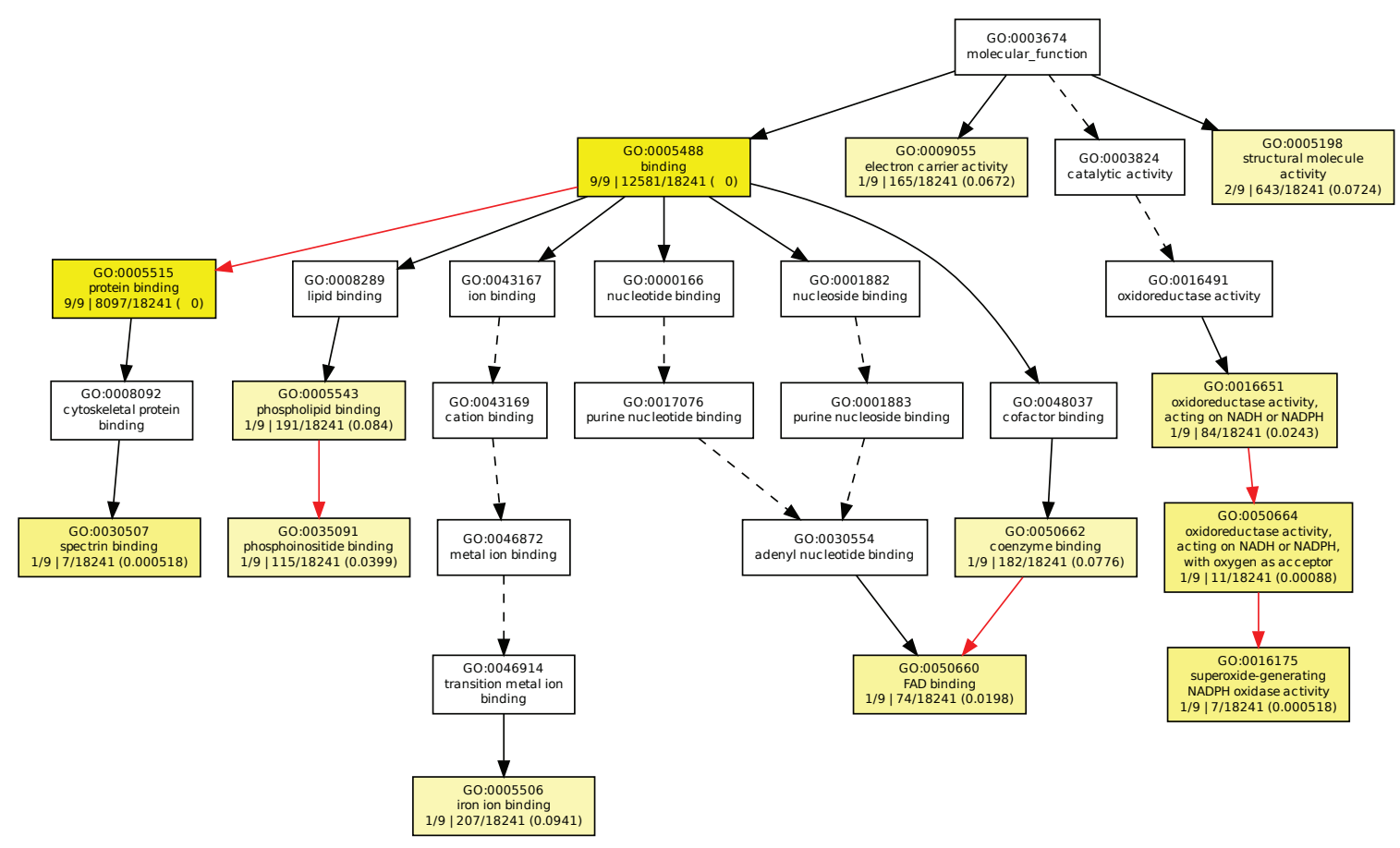

Figure 5 GOEAST analysis in molecular function category of T1D study. The GOEAST graphical output of enriched GO terms in the molecular function category for our selected gene network of T1D study. Boxes represent GO terms, labeled by its GO ID, term definition, and detailed information, organized as " $q / m \mid t / k(p-v a / u e)$ ", where $q$ is the count of genes associated with the listed GOID (directly or indirectly) in our dataset, $m$ is the count of genes associated with the listed GOID (directly or indirectly) on the chosen platform, $k$ is the total number of genes in our dataset, $t$ is the total number of genes on the chosen platform, $p$-value is of the significance for the enrichment in the dataset of the listed GOID under hypergeometric distribution. Significantly enriched GO terms are marked yellow. The degree of color saturation of each node is positively correlated with the enrichment significance of the corresponding GO term. Nonsignificant GO terms within the hierarchical tree are shown as white boxes. Branches of the GO hierarchical tree without significantly enriched GO terms are not shown. Arrows represent connections between different $\mathrm{GO}$ terms. Red arrows represent relationships between two enriched GO terms, black solid arrows represent relationships between enriched and unenriched terms and black dashed arrows represent relationships between two unenriched $\mathrm{GO}$ terms.

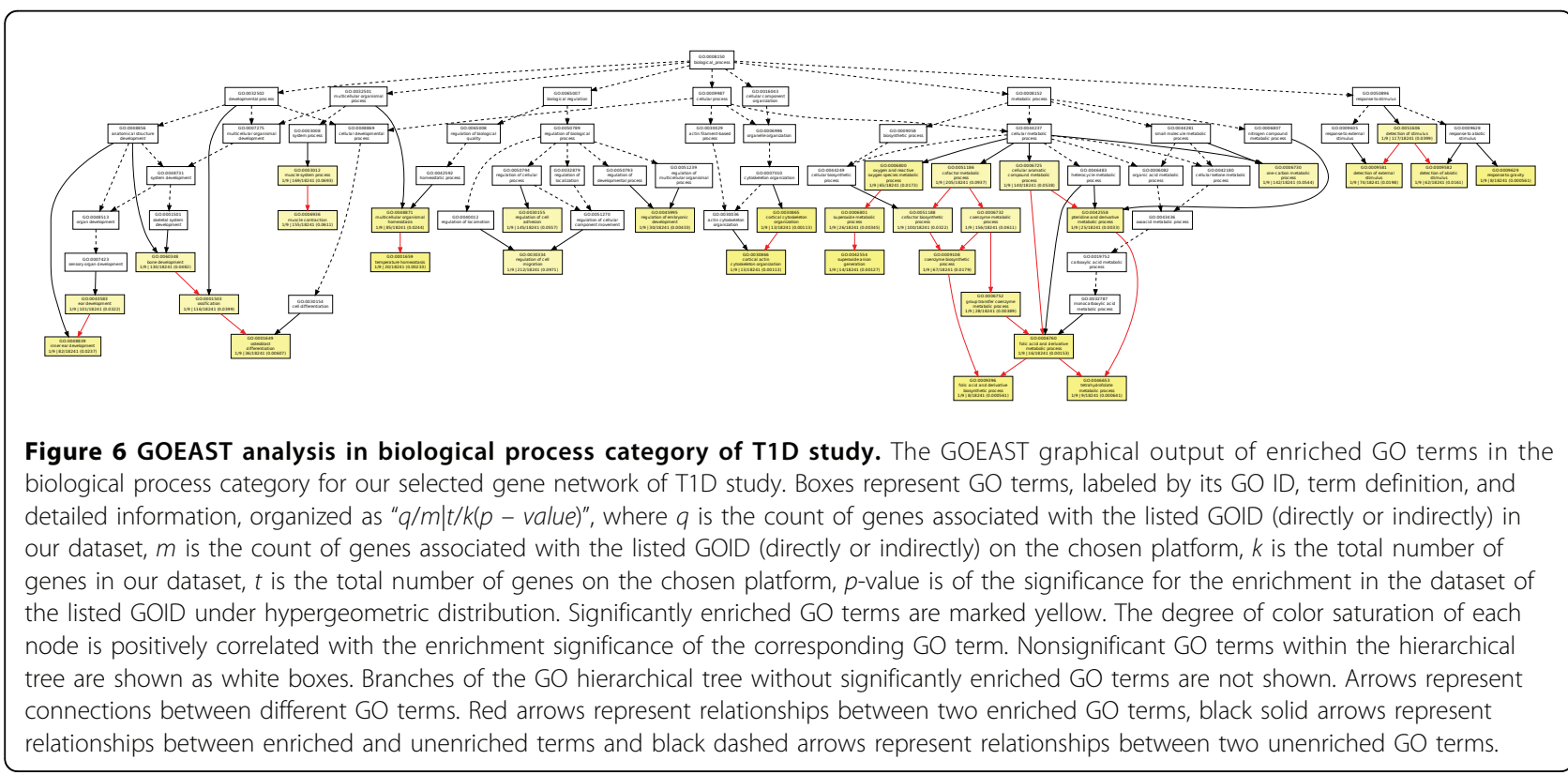




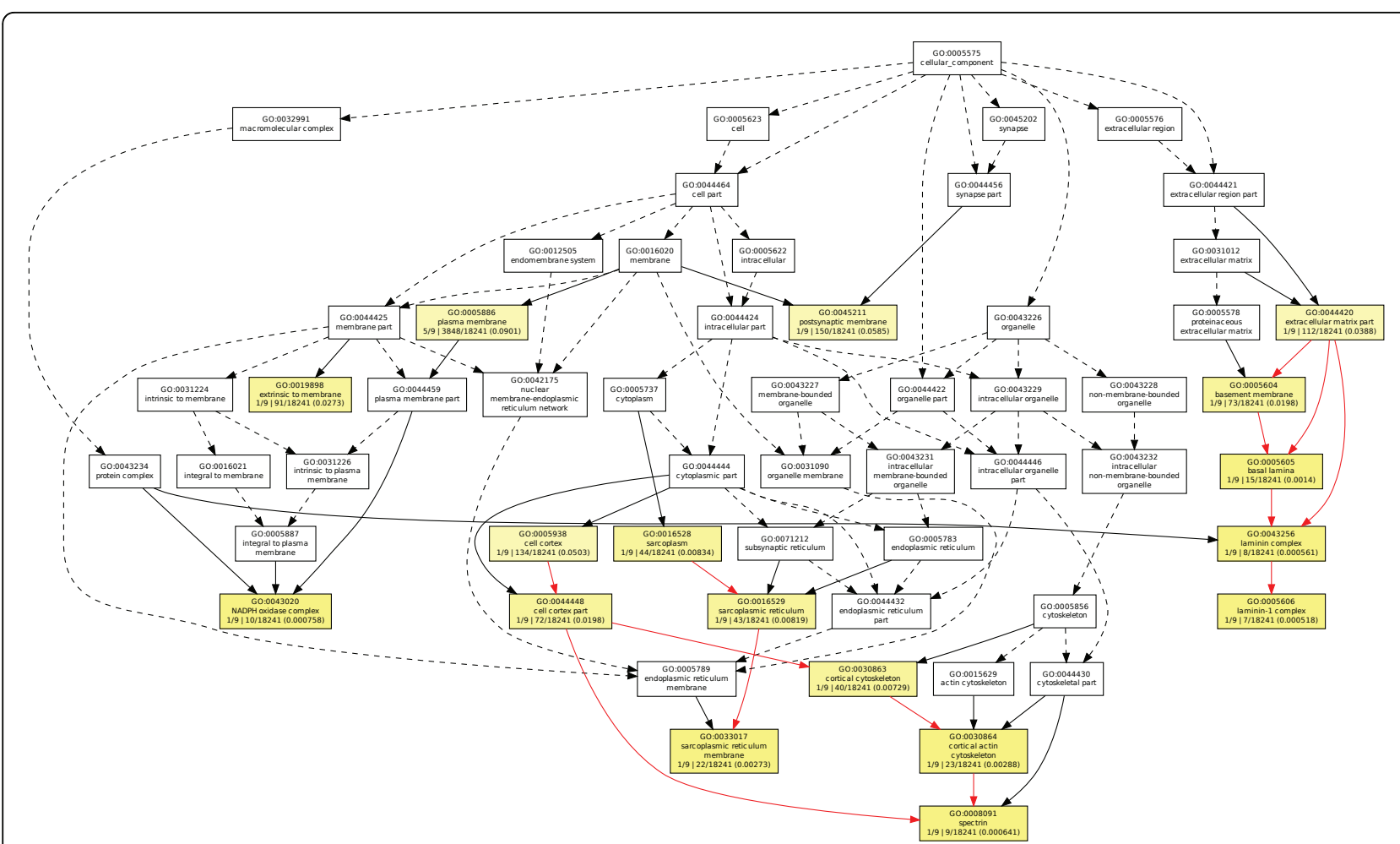

Figure 7 GOEAST analysis in cellular component category of T1D study. The GOEAST graphical output of enriched GO terms in the cellular component category for our selected gene network of T1D study. Boxes represent GO terms, labeled by its GO ID, term definition, and detailed information, organized as " $q / m \mid t / k(p$ - value)", where $q$ is the count of genes associated with the listed GOID (directly or indirectly) in our dataset, $m$ is the count of genes associated with the listed GOID (directly or indirectly) on the chosen platform, $k$ is the total number of genes in our dataset, $t$ is the total number of genes on the chosen platform, $p$-value is of the significance for the enrichment in the dataset of the listed GOID under hypergeometric distribution. Significantly enriched GO terms are marked yellow. The degree of color saturation of each node is positively correlated with the enrichment significance of the corresponding GO term. Nonsignificant GO terms within the hierarchical tree are shown as white boxes. Branches of the GO hierarchical tree without significantly enriched GO terms are not shown. Arrows represent connections between different GO terms. Red arrows represent relationships between two enriched GO terms, black solid arrows represent relationships between enriched and unenriched terms and black dashed arrows represent relationships between two unenriched GO terms.

Table 6 Gene Ontology terms significant associated with selected genes $(P<0.001)$ of T1D study.

\begin{tabular}{|c|c|c|c|c|}
\hline GO Category & GO ID & GO Annotation & Genes & $P$-value \\
\hline Molecular Function & GO:0016175 & superoxide-generating NADPH oxidase activity & NOX3 & 0.000518 \\
\hline Molecular Function & GO:0030507 & spectrin binding & EPB41L2 & 0.000518 \\
\hline Cellular Component & GO:0005606 & laminin-1 complex & LAMA2 & 0.000518 \\
\hline Biological Process & GO:0009629 & response to gravity & NOX3 & 0.000561 \\
\hline Biological Process & GO:0009396 & folic acid and derivative biosynthetic process & MTHFD1L & 0.000561 \\
\hline Cellular Component & GO:0043256 & laminin complex & LAMA2 & 0.000561 \\
\hline Biological Process & GO:0046653 & tetrahydrofolate metabolic process & MTHFD1L & 0.000641 \\
\hline Cellular Component & GO:0008091 & spectrin & EPB41L2 & 0.000641 \\
\hline Cellular Component & GO:0043020 & NADPH oxidase complex & NOX3 & 0.000758 \\
\hline Molecular Function & GO:0050664 & oxidoreductase activity, acting on NADH or NADPH, with oxygen as acceptor & NOX3 & 0.000880 \\
\hline
\end{tabular}


This data set consists of 330 controls and 121 cases of Chinese ethnicity. In this analysis, we excluded the centromeric and telomeric regions using $500 \mathrm{~kb}$ threshold because these regions tend to have bias in CNV calling. We transformed the probe intensities into LRR values from this SNP data set $(n=3369)$ using PennCNV with the same parameter setting and procedure as mentioned above.

\section{Classification results}

After a 10 -fold cross validation, 406 samples were selected as the training set and the remaining 45 samples were used as the testing set. Figure 8 illustrated the relationship of $\Delta$ and accuracy for our method. Our method achieved the optimal accuracy of $84.44 \%$, when the threshold of $\Delta$ was set either between $0.2-0.4$ or $0.6-3.1$. We showed all the 6 SNPs selected when $\Delta$ is

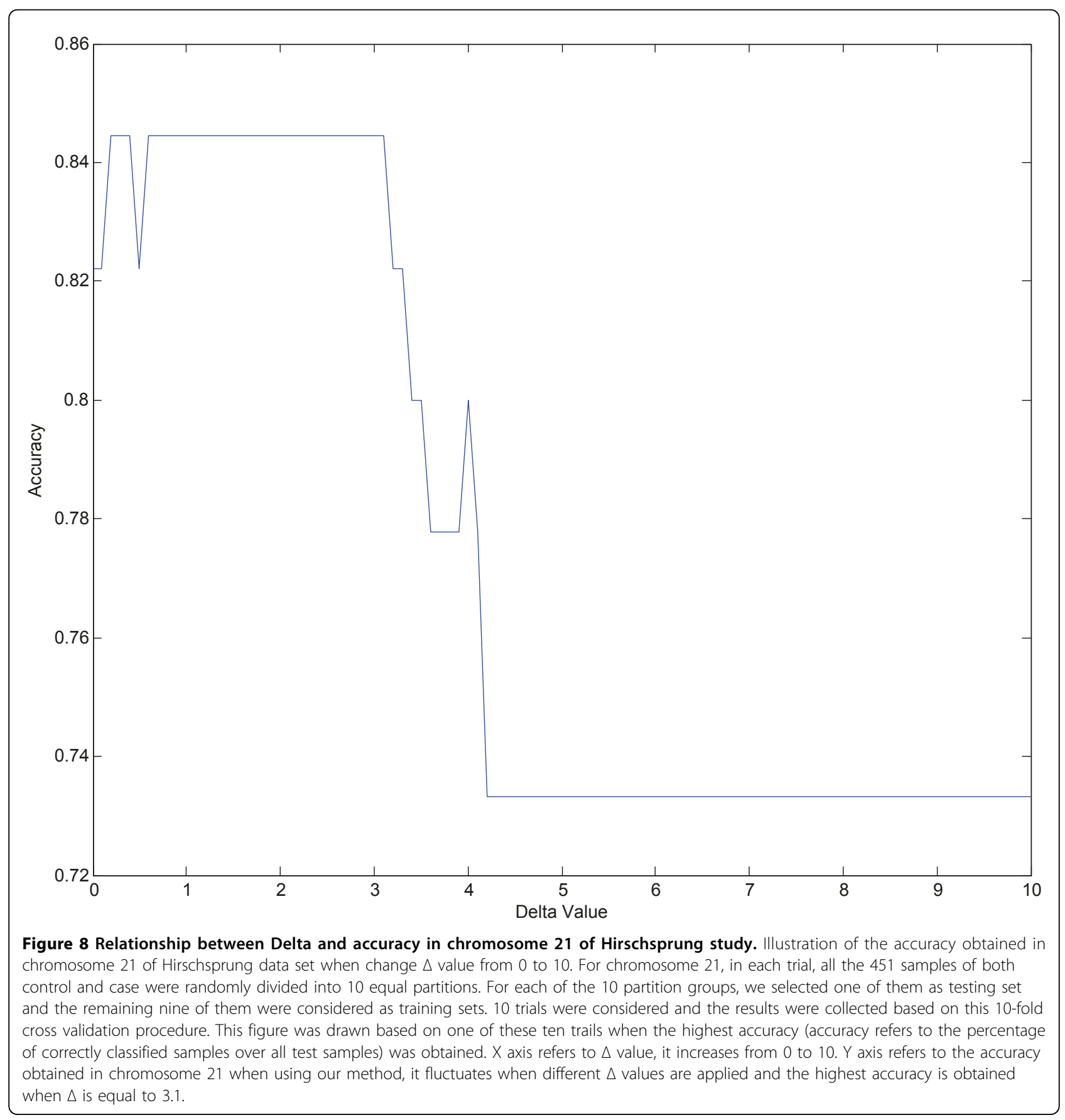


Table 7 Distribution of LRR values for selected SNPs of Hirschsprung study.

\begin{tabular}{llcccc}
\hline SNP ID & $d_{i k}$ & \multicolumn{2}{c}{ Control } & \multicolumn{2}{c}{ Case } \\
& & mean & variance & mean & variance \\
\hline rs11701130 & 1.0256 & -0.1325 & 0.1130 & 0.1821 & 0.0686 \\
rs2837770 & 0.4607 & -0.1387 & 0.1014 & 0.1208 & 0.0578 \\
rs2824050 & 0.3089 & -0.0559 & 0.0331 & 0.1185 & 0.0214 \\
rs928862 & 0.2301 & -0.0934 & 0.0632 & 0.1193 & 0.0339 \\
rs2824724 & 0.2177 & -0.1012 & 0.1169 & 0.1439 & 0.0626 \\
rs845930 & 0.1205 & -0.1543 & 0.2580 & 0.1668 & 0.1373 \\
\hline
\end{tabular}

equal to 3.1 in Table 7, it includes the average and variance of LRR values in each SNP position within case and control group respectively.

As there are only 6 SNPs selected, the number is too small to construct any networks. So for this dataset, we did not do any analysis in SNP level.

Gene network construction and analysis

All the 6 SNPs selected by our method belong to 3 unique genes. The detailed gene information can be seen in Table 8 . We calculated all the pairwise gene similarity values with the same parameter settings of previous data set. If define a threshold of 0.01 , all these 3 genes can be linked together, see Figure 9. After a survey in the literature about the known findings of these 3 genes, we found some interesting phenomena. For gene DSCAM, which is a previously implicated gene for the involvement of developing hirschsprung disease $[41,42]$, and for gene TMPRSS15, whose mutations can cause enterokinase deficiency, a malabsorption disorder characterized by diarrhea and failure to thrive, both of these two genes have a biological record that directly to hirschsprung disease, and both of them can be selected as relevant features using our method, furthermore, can be grouped together in our gene network, which can strongly prove the efficiency of our model.

For the gene network shown in Figure 9, the same procedure of enrichment analysis using GOEAST was

Table 8 Detailed descriptions of SNPs selected in chromosome 21 of Hirschsprung study.

\begin{tabular}{|c|c|c|c|}
\hline SNP ID & $\begin{array}{l}\text { Gene } \\
\text { Symbol }\end{array}$ & $\begin{array}{l}\text { Gene } \\
\text { ID }\end{array}$ & Description \\
\hline rs11701130 & intergenic & & \\
\hline rs2837770 & DSCAM & 1826 & $\begin{array}{l}\text { Down syndrome cell adhesion } \\
\text { molecule }\end{array}$ \\
\hline rs2824050 & intergenic & & \\
\hline rs928862 & intergenic & & \\
\hline rs2824724 & TMPRSS15 & 5651 & $\begin{array}{l}\text { Mutations in this gene cause } \\
\text { enterokinase deficiency, a } \\
\text { malabsorption disorder characterized } \\
\text { by diarrhea and failure to thrive }\end{array}$ \\
\hline rs845930 & TIAM1 & 7074 & $\begin{array}{l}\text { T-cell lymphoma invasion and } \\
\text { metastasis } 1\end{array}$ \\
\hline
\end{tabular}

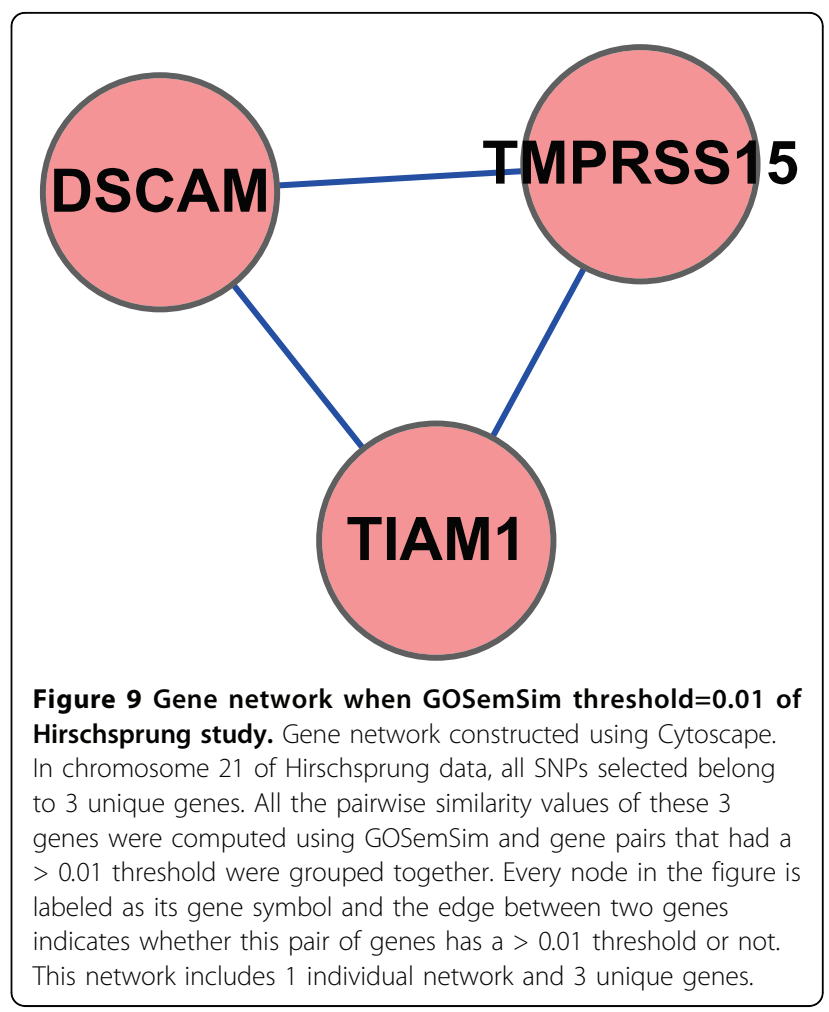

done, see Figures 10, 11, 12. Table 9 shows a detailed description of GO terms with a significant $P<0.0001$, which can provide an inner functions of our selected genes.

\section{Conclusions}

In this paper, we use the method of nearest shrunken centroid for gene expression data, and apply it to tackle copy number variation data determined from genomewide SNP arrays. The method can be implemented on a personal computer very efficiently. The relevant SNPs are selected for disease data. Experimental results are reported to show the effectiveness of our method. In particular, we find some SNPs that contain in some genes which are relevant to a particular disease. Based on the SNP and gene networks, we can find out some unknown relationships between their corresponding genes, which can be considered as an extension of existing GO knowledge. The existing Gene Ontology enrichment analysis tool also suggests that our selected genes are associated to some molecular function and biological process. In the future, we will study the following problems. Detailed biological analysis of CNVs determined from other genome-wide SNP data sets will be studied. Statistical and association study of selected SNPs can be carried out. 


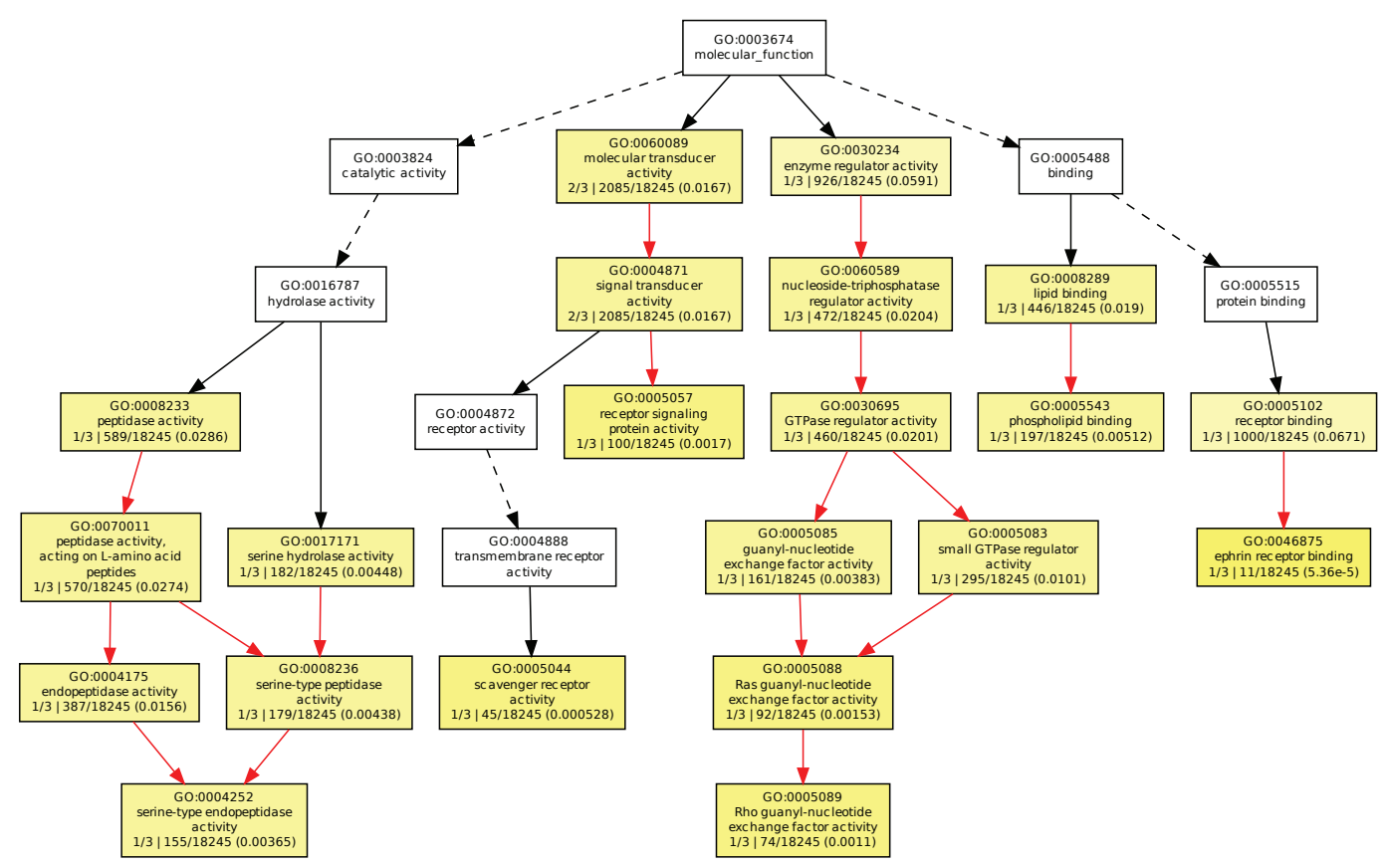

Figure 10 GOEAST analysis in molecular function category of Hirschsprung study. The GOEAST graphical output of enriched GO terms in the molecular function category for our selected gene network of Hirschsprung study. Boxes represent GO terms, labeled by its GO ID, term definition, and detailed information, organized as " $q / m \mid t / k(p$ - value)", where $q$ is the count of genes associated with the listed GOID (directly or indirectly) in our dataset, $m$ is the count of genes associated with the listed GOID (directly or indirectly) on the chosen platform, $k$ is the total number of genes in our dataset, $t$ is the total number of genes on the chosen platform, $p$-value is of the significance for the enrichment in the dataset of the listed GOID under hypergeometric distribution. Significantly enriched GO terms are marked yellow. The degree of color saturation of each node is positively correlated with the enrichment significance of the corresponding GO term. Nonsignificant GO terms within the hierarchical tree are shown as white boxes. Branches of the GO hierarchical tree without significantly enriched GO terms are not shown. Arrows represent connections between different GO terms. Red arrows represent relationships between two enriched GO terms, black solid arrows represent relationships between enriched and unenriched terms and black dashed arrows represent relationships between two unenriched GO terms.

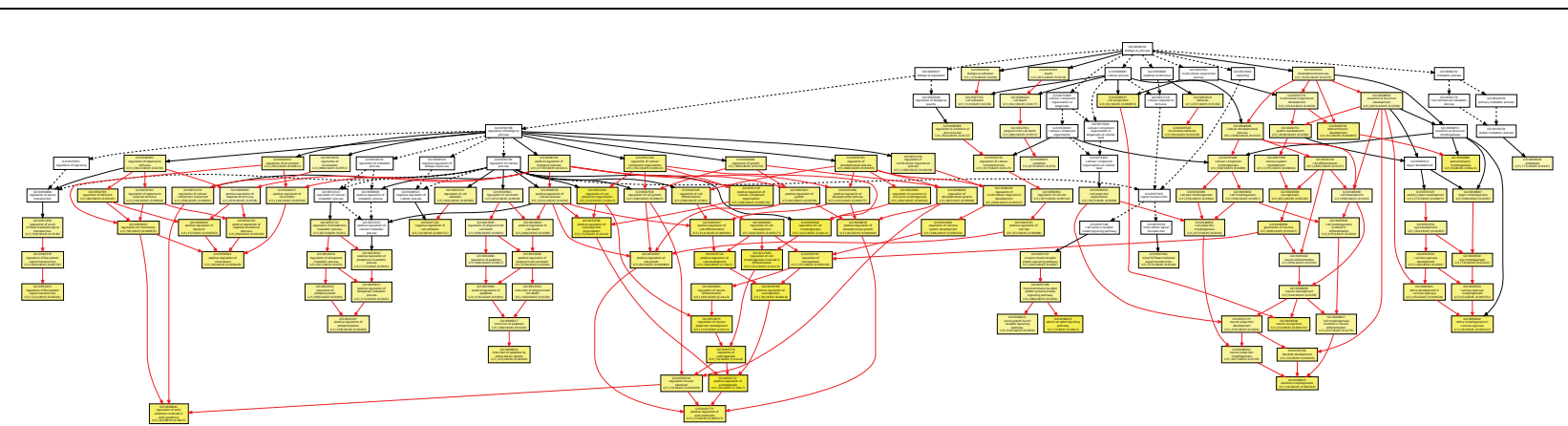

Figure 11 GOEAST analysis in biological process category of Hirschsprung study. The GOEAST graphical output of enriched GO terms in the biological process category for our selected gene network of Hirschsprung study. Boxes represent GO terms, labeled by its GO ID, term definition, and detailed information, organized as " $q / \mathrm{m} \mid t / k(p$ - value)", where $q$ is the count of genes associated with the listed GOID (directly or indirectly) in our dataset, $m$ is the count of genes associated with the listed GOID (directly or indirectly) on the chosen platform, $k$ is the total number of genes in our dataset, $t$ is the total number of genes on the chosen platform, $p$-value is of the significance for the enrichment in the dataset of the listed GOID under hypergeometric distribution. Significantly enriched GO terms are marked yellow. The degree of color saturation of each node is positively correlated with the enrichment significance of the corresponding GO term. Nonsignificant GO terms within the hierarchical tree are shown as white boxes. Branches of the GO hierarchical tree without significantly enriched GO terms are not shown. Arrows represent connections between different GO terms. Red arrows represent relationships between two enriched GO terms, black solid arrows represent relationships between enriched and unenriched terms and black dashed arrows represent relationships between two unenriched GO terms. 


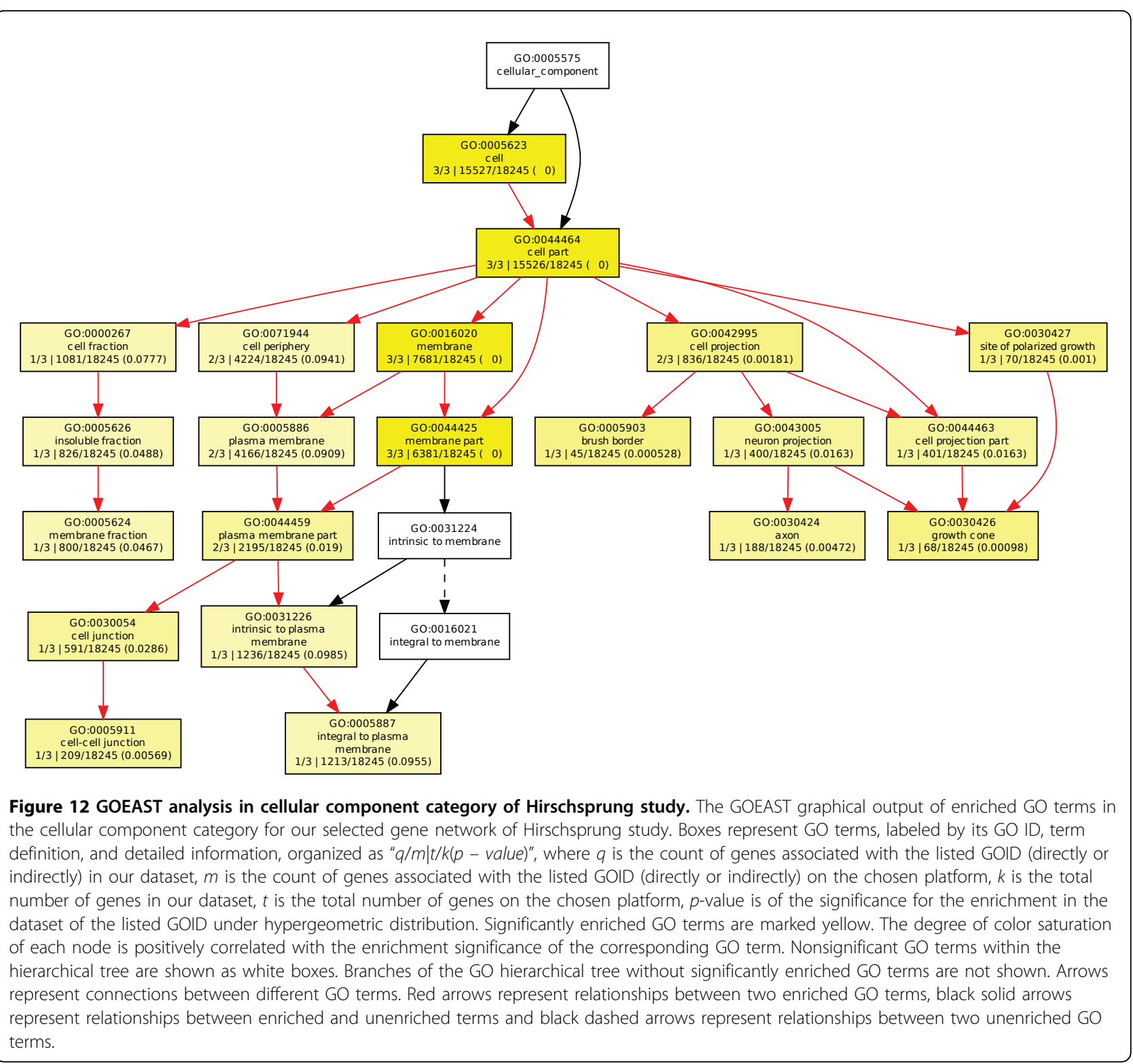

Table 9 Gene Ontology terms significant associated with selected genes $(P<0.0001)$ of Hirschsprung study.

\begin{tabular}{lllll}
\hline GO Category & GO ID & GO Annotation & Genes & P-value \\
\hline Biological Process & GO:0050772 & positive regulation of axonogenesis & DSCAM, TIAM1 & 0.000000736 \\
Biological Process & GO:0031346 & positive regulation of cell projection organization & DSCAM, TIAM1 & 0.000005400 \\
Biological Process & GO:0048013 & ephrin receptor signaling pathway & TIAM1 & 0.000024800 \\
Biological Process & GO:0031344 & regulation of cell projection organization & DSCAM, TIAM1 & 0.000030600 \\
Biological Process & GO:0009886 & post-embryonic morphogenesis & DSCAM & 0.000038400 \\
Biological Process & GO:0048841 & regulation of axon extension involved in axon guidance & DSCAM & 0.000045900 \\
Molecular Function & GO:0046875 & ephrin receptor binding & TIAM1 & 0.000053600 \\
\hline
\end{tabular}




\section{Authors contributions}

MN designed this study and developed the new algorithm. YL designed this study, coded the program, ran the experiments and wrote the manuscript. YF designed this study.

\begin{abstract}
Acknowledgements
This work was financially supported by Research Grant Council [201508] and Hong Kong Baptist University FRGs.

The authors would like to extend gratitude to the support by research grants, including HKU 765407M and HKU 775907M from the Hong Kong Research Grants Council, AOSPINE (AOSBRC-07-02) and AoE/M-04/04 from the University Grants Committee of Hong Kong. We are also grateful to Prof. Kathryn S.E. Cheah, Prof. Pak C. Sham, Prof. Paul K.H. Tam, Dr Maria-Merc, Garcia-Barcel, Dr YQ Song, Dr Danny Chan, Dr Kenneth Cheung and Dr Clara S. Tang for their assistance and kindness in data sharing.

This article has been published as part of BMC Bioinformatics Volume 12 Supplement 5, 2011: Selected articles from the IEEE International Conference on Bioinformatics and Biomedicine 2010. The full contents of the supplement are available online at http://www.biomedcentral.com/14712105/12?issue $=$ S5.
\end{abstract}

\section{Competing interests}

The authors declare that they have no competing interests.

Published: 27 July 2011

\section{References}

1. Feuk $L$, Carson AR, Scherer SW: Structural variation in the human genome. Nature Reviews Genetics 2006, 7:85-97.

2. Freeman JL, Perry GH, Feuk L, Redon R, McCarroll SA, Altshuler DM Aburatani H, Jones KW, Tyler-Smith C, Hurles ME, Carter NP, Scherer SW, Lee C: Copy number variation: New insights in genome diversity. Genome Research 2006, 16:949-961.

3. Sebat J, Lakshmi B, Troge J, Alexander J, Young J, Lundin P, Maner S, Massa H, Walker M, Chi M, Navin N, Lucito R, Healy J, Hicks J, Ye K, Reiner A, Gilliam TC, Trask B, Patterson N, Zetterberg A, Wigler M: Large-Scale Copy Number Polymorphism in the Human Genome. Science 2004, 305:525-528.

4. lafrate AJ, Feuk L, Riveral MN, Listewnik ML, Donahoe PK, Qi Y, Scherer SW, Lee C: Detection of large-scale variation in the human genome. Nature Genetics 2004, 36:949-951.

5. Conrad DF, Andrews TD, Carter NP, Hurles ME, Pritchard JK: A highresolution survey of deletion polymorphism in the human genome. Nature Genetics 2006, 38:75-81.

6. McCarroll SA, Hadnott TN, Perry GH, Sabeti PC, Zody MC, Barrett JC, Dallaire S, Gabriel SB, Lee C, Daly MJ, Altshuler DM: Common deletion polymorphisms in the human genome. Nature Genetics 2006, 38:86-92.

7. Redon R, Ishikawa S, Fitch KR, Feuk L, Perry GH, Andrews TD, Fiegler H, Shapero MH, Carson AR, Chen W, Cho EK, Dallaire S, Freeman JL, Gonzalez JR, Gratacos M, Huang J, Kalaitzopoulos D, Komura D, MacDonald JR, Marshall CR, Mei R, Montgomery L, Nishimura K, Okamura K, Shen F, Somerville MJ, Tchinda J, Valsesia A, Woodwark C, Yang F, Zhang J, Zerjal T, Zhang J, Armengol L, Conrad DF, Estivill X, Tyler-Smith C, Carter NP, Aburatani H, Lee C, Jones KW, Scherer SW, Hurles ME: Global variation in copy number in the human genome. Nature 2006, 444:444-454.

8. Kallioniemi A, Kallioniemi OP, Sudar D, Rutovitz D, Gray JW, Waldman F, Pinkel D: Comparative genomic hybridization for molecular cytogenetic analysis of solid tumors. Science 1992, 258:818-821.

9. Pinkel D, Segraves R, Sudar D, Clark S, Poole I, Kowbel D, Collins C, Kuo WL, Chen C, Zhai Y, Dairkee SH, Ljung B, Gray JW, Albertson DG: High resolution analysis of DNA copy number variation using comparative genomic hybridization to microarrays. Nature Genetics 1998, 20:207-211.

10. Wang P, Kim Y, Pollack J, Narasimhan B, Tibshirani R: A method for calling gains and losses in array CGH data. Biostatistics 2005, 6:45-58.
11. Pique-Regi R, Monso-Varona J, Ortega A, Seeger RC, Triche TJ, Asgharzadeh S: Sparse representation and Bayesian detection of genome copy number alterations from microarray data. Bioinformatics 2008, 24(3):309-318.

12. Wu LY, Chipman HA, Bull SB, Briollais L, Wang K: A Bayesian segmentation approach to ascertain copy number variations at the population level. Bioinformatics 2009, 25(13):1669-1679.

13. Chen J, Wang YP: A Statistical Change Point Model Approach for the Detection of DNA Copy Number Variations in Array CGH Data. IEEE/ACM Transactions on Computational Biology and Bioinformatics 2009, 6:529-541.

14. Oldridge DA, Banerjee S, Setlur SR, Sboner A, Demichelis F: Optimizing copy number variation analysis using genome-wide short sequence oligonucleotide arrays. Nucleic Acids Research 2010, 38:3275-3286.

15. Zhao X, Li C, Paez JG, Chin K, Janne PA, Chen T, Girard L, Minna J, Christiani D, Leo C, Gray JW, Sellers WR, Meyerson M: An Integrated View of Copy Number and Allelic Alterations in the Cancer Genome Using Single Nucleotide Polymorphism Arrays. Cancer Research 2004, 64:3060-3071.

16. Huang J, Wei W, Zhang J, Liu G, Bignell GR, Stratton MR, Futreal PA, Wooster R, Jones KW, Shapero MH: Whole genome DNA copy number changes identified by high density oligonucleotide arrays. Hum Genomics 2004, 1:287-299.

17. Colella S, Yau C, Taylor JM, Mirza G, Butler H, Clouston P, Bassett AS, Seller A, Holmes CC, Ragoussis J: QuantiSNP: an Objective Bayes HiddenMarkov Model to detect and accurately map copy number variation using SNP genotyping data. Nucleic Acids Research 2007, 35:2013-2025.

18. Wang K, Li M, Hadley D, Liu R, Glessner J, Grant SFA, Hakonarson H, Bucan M: Penn CNV: An integrated hidden Markov model designed for high-resolution copy number variation detection in whole-genome SNP genotyping data. Genome Research 2007, 17:1665-1674.

19. Bastian BC, Olshen AB, LeBoit PE, Pinkel D: Classifying Melanocytic Tumors Based on DNA Copy Number Changes. American Journal of Pathology 2003, 163:1765-1770

20. O'Hagan RC, Brennan CW, Strahs A, Zhang X, Kannan K, Donovan M, Cauwels C, Sharpless NE, Wong WH, Chin L: Array Comparative Genome Hybridization for Tumor Classification and Gene Discovery in Mouse Models of Malignant Melanoma. Cancer Res 2003, 63:5352-5356.

21. Baumbusch LO, Aaroe J, Johansen FE, Hicks J, Sun H, Bruhn L, Gunderson K, Naume B, Kristensen VN, Liestol K, Borresen-Dale AL, Lingjaerde OC: Comparison of the Agilent, ROMA/NimbleGen and Illumina platforms for classification of copy number alterations in human breast tumors. BMC Genomics 2008, 9:379.

22. Myllykangas S, Tikka J, Bohling T, Knuutila S, Hollmen J: Classification of human cancers based on DNA copy number amplification modeling. BMC Medical Genomics 2008, 1:15.

23. Korn JM, Kuruvilla FG, McCarroll SA, Wysoker A, Nemesh J, Cawley S, Hubbell E, Veitch J, Collins PJ, Darvishi K, Lee C, Nizzari MM, Gabriel SB, Purcell S, Daly MJ, Altshuler D: Integrated genotype calling and association analysis of SNPs, common copy number polymorphisms and rare CNVs. Nature Genetics 2008, 40:1253-1260.

24. Nannya Y, Sanada M, Nakazaki K, Hosoya N, Wang L, Hangaishi A, Kurokawa M, Chiba S, Bailey DK, Kennedy GC, Ogawa S: A Robust Algorithm for Copy Number Detection Using High-Density Oligonucleotide Single Nucleotide Polymorphism Genotyping Arrays. Cancer Res 2005, 65:6071-6079.

25. Li C, Wong WH: DNA-Chip Analyzer (dChip). In The analysis of gene expression data: methods and software. New York: Springer;Parmigiani $G$, Garrett ES, Irizarry R, Zeger SL 2003:120-141.

26. Lin M, Wei LJ, Sellers WR, Lieberfarb M, Wong WH, Li C: dChipSNP: Significance Curve and Clustering of SNP-Array-Based Loss-ofHeterozygosity Data. Bioinformatics 2004, 20:1233-1240.

27. Hupe P, Stransky N, Thiery J, Radvanyi F, Barillot E: Analysis of array CGH data: from signal ratio to gain and loss of DNA regions. Bioinformatics 2004, 20:3413-3422.

28. Peiffer DA, Le JM, Steemers FJ, Chang W, Jenniges T, Garcia F, Haden K, Li J, Shaw CA, Belmont J, Cheung SW, Shen RM, Barker DL, Gunderson KL: Highresolution genomic profiling of chromosomal aberrations using Infinium whole-genome genotyping. Genome Res 2006, 16(9):1136-1148.

29. Bair E, Tibshirani R: Machine learning method applied to DNA microarray data can improve the diagnosis of cancer. S/GKDD Explorations 2003, 5:48-55. 
30. Tibshirani R, Hastie T, Narasimhan B, Chu G: Diagnosis of multiple cancer types by shrunken centroids of gene expression. Proc Natl Acad Sci U S A. 2002, 99(10):6567-6572.

31. PLINK. [http://pngu.mgh.harvard.edu/purcell/plink].

32. Burton P: Genome-wide association study of 14,000 cases of seven common diseases and 3,000 shared controls. Nature 2007, 447:661-678.

33. Hovind P, Hansen TK, Tarnow L, Thiel S, Steffensen R, Flyvbjerg A, Parving H: Mannose-Binding Lectin as a Predictor of Microalbuminuria in Type 1 Diabetes. Diabetes 2005, 54:1523-1527.

34. Maassen A, 't Hart LM, van Essen E, Heine RJ, Nijpels G, Tafrechi RSJ, Raap AK, Janssen GMC, Lemkes HH: Mitochondrial Diabetes: Molecular Mechanisms and Clinical Presentation. Diabetes 2004, 53:S103-S109.

35. Tarsio JF, Reger LA, Furcht LT: Molecular mechanisms in basement membrane complications of diabetes. Alterations in heparin, laminin, and type IV collagen association. Diabetes 1988, 37:532-539.

36. Bioconductor. [http://www.bioconductor.org].

37. Ashburner M, Ball CA, Blake JA, Botstein D, Butler H, Cherry JM, Davis AP, Dolinski K, Dwight SS, Eppig JT, Harris MA, Hill DP, Issel-Tarver L, Kasarskis A, Lewis S, Matese JC, Richardson JE, Ringwald M, Rubin GM, Sherlock G: Gene ontology: tool for the unification of biology. Nat Genet 2000, 25:25-29.

38. Schlicker A, Domingues FS, Rahnenfuhrer J, Lengauer T: A new measure for functional similarity of gene products based on Gene Ontology. BMC Bioinformatics 2006, 7:302.1-302.16.

39. Shannon P, Markiel A, Ozier O, Baliga NS, Wang JT, Ramage D, Amin N, Schwikowski B, Ideker T: Cytoscape: a software environment for integrated models of biomolecular interaction networks. Genome Res 2003, 13:2498-2504.

40. Zheng Q, Wang XJ: GOEAST: a web-based software toolkit for Gene Ontology enrichment analysis. Nucleic Acids Res 2008, 36(Web Server issue):W358-363.

41. Korbel JO, Tirosh-Wagner T, Urban AE, Chen XN, Kasowski M, Dai L, Grubert F, Erdman C, Gao MC, Lange K, Sobel EM, Barlow GM, Aylsworth AS, Carpenter NJ, Clark RD, Cohen MY, Doran E, Falik-Zaccai T, Lewin SO, Lott IT, McGillivray BC, Moeschler JB, Pettenati MJ, Pueschel SM, Rao KW, Shaffer LG, Shohat M, Riper AJV, Warburton D, Weissman S, Gerstein MB, Snyder M, Korenberg JR: The genetic architecture of Down syndrome phenotypes revealed by high-resolution analysis of human segmental trisomies. PNAS 2009, 106:12031-12036.

42. Yamakawa K, Huot YK, Haendelt MA, Hubert R, Chen XN, Lyons GE, Korenberg JR: DSCAM: a novel member of the immunoglobulin superfamily maps in a Down syndrome region and is involved in the development of the nervous system. Human Molecular Genetics 1998, 7:227-237.

doi:10.1186/1471-2105-12-S5-S4

Cite this article as: Liu et al:: SNP and gene networks construction and analysis from classification of copy number variations data. BMC Bioinformatics 2011 12(Suppl 5):S4.

\section{Submit your next manuscript to BioMed Central and take full advantage of:}

- Convenient online submission

- Thorough peer review

- No space constraints or color figure charges

- Immediate publication on acceptance

- Inclusion in PubMed, CAS, Scopus and Google Scholar

- Research which is freely available for redistribution

Submit your manuscript at www.biomedcentral.com/submit
Biomed Central 